\title{
Littérature et image : d'une lecture à l'autre
}

\section{Françoise Demougin}

\section{(2) OpenEdition}

Journals

Édition électronique

URL : http://journals.openedition.org/trema/1584

DOI : 10.4000/trema.1584

ISSN : 2107-0997

\section{Éditeur}

Faculté d'Éducation de l'université de Montpellier

\section{Édition imprimée}

Date de publication : 1 octobre 2002

Pagination : 55-66

ISSN : 1167-315X

\section{Référence électronique}

Françoise Demougin, « Littérature et image : d'une lecture à l'autre », Tréma [En ligne], 19 | 2002, mis en ligne le 23 janvier 2013, consulté le 10 décembre 2020. URL : http://journals.openedition.org/trema/ 1584 ; DOl : https://doi.org/10.4000/trema.1584

Ce document a été généré automatiquement le 10 décembre 2020.

Trema 


\title{
Littérature et image : d'une lecture à l'autre
}

\author{
Françoise Demougin
}

1 L'intégration de l'image à l'école, puis au collège et au lycée dans le cours de français, a débuté il y a bien longtemps, avec les vieux abécédaires du XIXe siècle. L'image à l'école est apparue d'ailleurs sous des formes très diverses: gravures en noir et blanc, puis en couleurs, films fixes projetés, photographies, dessins, reproductions de photogrammes, de tableaux... La nouveauté vient cependant aujourd'hui de sa promotion au rang d'objet d'étude et d'enseignement. L'image est devenue une catégorie à part entière des programmes de $1995^{1}$, et en 1998 le terme de « lecture de l'image » a été introduit dans les textes officiels. Les manuels récents se font l'écho de l'apparition de la lecture de l'image dans les pratiques réelles des enseignants. L'« éducation à l'image» est la première des «nouvelles actions prioritaires» indiquées dans le dossier de presse "Partenariats Éducation - Culture " : l'image se trouve inscrite dans le cadre de « la maîtrise du langage et la sensibilisation aux arts ». Intégrée ainsi dans le cadre de la maîtrise du langage, l'image fait naturellement partie des programmes de français du collège - depuis 1987 - placée toutefois sous le signe de la relation texte - image. Une évolution récente (décelable notamment à travers les projets sur les nouveaux programmes de l'école primaire, soumis à consultation nationale depuis août 2001) fait hésiter l'image entre le champ disciplinaire auquel on la rattache (celui du français) et une transversalité relevant de sa spécificité communicationnelle. Ainsi, en cycle 3 du primaire, l'image peut-elle relever à la fois de «la maîtrise du langage» - écrit ou oral - de «l'éducation littéraire et humaine ", et de «l'observation réfléchie de la langue française », selon les trois termes retenus officiellement pour le primaire.

2 On peut s'interroger sur les incidences de cette «entrée en image » quasi religieuse de l'école, de l'enseignant, de l'enseignant de lettres en particulier. Nous voudrions ici analyser trois conséquences qui pour n'être pas exhaustives n'en sont pas moins à notre sens importantes et révélatrices des changements forcément opérés à travers l'utilisation d'un matériau didactique neuf. 


\section{De nouveaux objets d'étude}

3 L'image se trouve au centre d'une série de contradictions qui la rendent particulièrement intéressante : si elle paraît, le plus souvent, dénuée de légitimité dans le champ littéraire, elle tend à devenir un objet d'étude légitime, dans le champ scolaire, par son rapport au texte écrit et littéraire, en particulier. Trois phénomènes ont contribué à cette évolution. Tout d'abord le domaine de l'image s'est détaché de la sphère ludique pour migrer vers le champ littéraire (dans le même temps d'ailleurs où la littérature de jeunesse se détachait de la sphère éducative pour aller vers ce même champ). En réalité, un conflit de représentations s'est installé, à propos de l'image, qui reste ancrée dans le ludique pour partie et, pour une autre partie, participe de la création artistique : double postulation que l'école se doit d'assumer.

4 L'intégration ensuite dans les théories de la lecture de diverses approches de la « réception » a amené une prise en compte du lecteur - spectateur comme lecteur expert, alors que le caractère global et « universel » de l'image implique une lecture spontanée, naïve, projective. Enfin la didactique de la lecture a évolué, influencée par les deux phénomènes que nous venons de souligner. Que ce soit en termes de problématiques de recherches, d'institutions d'accueil, de réseaux ou d'instances universitaires (par les « sections » au sein du Conseil national des Universités), l'image émarge en somme à trois domaines : la littérature, l'histoire de l'art et les sciences de l'éducation. La difficulté est alors de trouver un territoire stable pour cette image tant sollicitée. Les tentatives ont été nombreuses, pas forcément heureuses, en particulier dans des propositions qui faisaient la part belle à la littérature, au texte écrit.

5 Au-delà de l'image, fixe ou mobile, isolée ou en séquences, c'est la question du rapport texte - image qui se trouve fréquemment posée parce qu'elle cristallise les contradictions que nous venons de décrire. L'album de jeunesse, la bande dessinée, le film, le tableau sont devenus des supports de lecture et d'activités pédagogiques au même titre que le livre, que le texte écrit, ou à côté de celui-ci, à sa marge, et ils sont bien souvent saisis dans leur lien au texte, à l'écrit lisible. Ainsi l'album de jeunesse illustré fait désormais partie de la classe du primaire, pour les trois cycles puisqu'il peut s'adresser à des enfants qui ne savent pas lire comme à des lecteurs confirmés. Textes et images réunis y concourent à une proposition narrative qui invite les jeunes lecteurs à construire du sens, à réagir, à s'émouvoir, à se repérer dans la polysémie de la relation texte - image, tout cela étayé par un travail sur le paratexte, le texte et l'intertexte. Ce lien texte - image, si évident dans les pratiques scolaires, suppose quelques malentendus.

D'abord facilitatrice d'une lecture experte du texte écrit, l'image est ensuite devenue timidement objet d'une lecture spécifique avant de retourner vers le texte écrit dans un acte de lecture saisi gémellairement. Prenons l'exemple de la bande dessinée. De nombreux manuels y ont eu d'abord recours pour matérialiser des notions de narratologie dont la dimension abstraite empêchait une claire compréhension de la part des élèves. Ainsi en a-t-il été de la notion de focalisation empruntée à G. Genette. L'image ne servait alors que de réflecteur en quelque sorte pour la notion de point de vue. L'utilisation de l'image pour faire comprendre les outils de l'analyse littéraire est encore une pratique courante chez les enseignants, ravis de trouver ce qui leur apparaît comme un équivalent iconique bien pratique didactiquement. Certains vont plus loin, imputant à l'image cinématographique les nouvelles formes d'écriture romanesque moderne et 
contemporaine. Gageons pourtant que la littérature n'a pas attendu le cinéma pour saisir l'effet de sens de la description rapprochée d'un visage. Même les techniques nouvelles d'écriture romanesque, révolutionnaires par rapport à la tradition romanesque, d'un auteur comme Malraux, cinéphile averti, réalisateur lui-même d'un film Sierra de Teruel, ne peuvent s'expliquer par l'application de «recettes cinématographiques». Certes l'esthétique de la discontinuité, exhibée par la spatialité de l'image, a bouleversé la conception de l'espace et du temps dans l'écriture romanesque et des formes littéraires nouvelles sont apparues mais faut-il pour autant parler d'écriture cinématographique et tout expliquer par là ? Dans ces exemples, l'image est utilisée comme outil et modèle de conceptualisation dans le cadre de la lecture savante du texte littéraire. C'est la source d'un premier malentendu qui conduit à des simplifications et à des transferts abusifs dont le rendement didactique est faible, et parfois contre-productif.

Un deuxième usage de la bande dessinée, et donc de l'image, consiste à y trouver un accès simplifié à l'étude d'oeuvres littéraires réputées difficiles : ainsi étudier Proust au collège devient possible, comme le montre un récent travail proposé dans la revue Le français dans tous ses états. De la même manière, il semble que le cinéma, ou la télévision plus récemment, soit promue au rang de grands sauveurs des romans classiques du XIX ${ }^{\mathrm{e}}$ siècle 2. Féval, Dumas, Balzac, Hugo ont été réédités à la suite d'adaptations cinématographiques. Enfin la bande dessinée est étudiée aussi dans sa spécificité communicationnelle et esthétique, souvent d'ailleurs dans une perspective de production: les élèves sont amenés à élaborer eux-mêmes des bandes dessinées, des historiettes créées de toutes pièces avec ou sans canevas préalable. Tout se passe comme si le graphisme de l'image résolvait tout autre problème résultant de la mise en cohérence et cohésion d'un récit imaginé. De la même manière, l'écriture d'un scénario devient une activité rebattue. Dans ces différentes hypothèses, l'image est considérée comme outil de facilitation procédurale, pour l'acquisition d'une culture et d'un savoirfaire scriptural. Dans tous les cas, l'image est instrumentalisée.

8 Cet aspect salvateur de l'image (et presque rédempteur : hors de l'image point de salut...) prêterait seulement à sourires pour les uns, à grincements de dents pour les autres, devant la naïveté des vertus qu'on lui prête, si on n'y décelait, au delà des services qu'on en attend, à l'articulation entre le lisible et le visible, la base d'une véritable activité réflexive sur la lecture: le spectateur - lecteur de l'image, confronté aux exigences scolaires de la lecture, est conduit à en transférer certains usages dans la lecture d'images, à en mesurer l'inadéquation, bref à faire entrer en conflit un usage scolaire établi (la lecture, avec ses critères, ses finalités, comme la compréhension, ses démarches cognitives, par synthèses successives) et une pratique spontanée qui ne peut s'y résoudre totalement (il ne peut pas lire seulement de gauche à droite l'image, il ne se borne pas à la comprendre, le temps consacré à l'activité est moins linéaire...). Dans ce conflit entre une pratique scolaire portant sur l'écrit et une pratique privée portant sur l'iconique, se situe la possibilité d'une attitude réflexive sur l'une et l'autre, la nécessité d'un parcours heuristique propre à construire les outils spécifiques d'une lecture de l'image. Le nouvel objet d'étude qui se dessine, si l'on veut bien tirer les conséquences des nouveaux programmes, n'est pas tant l'image instrumentalisée au service de la lecture littéraire ou l'image étudiée en elle-même, mais bien l'activité de lecture de l'image comme distance réflexive par rapport à l'acte de lecture, comme passage d'une lecture (celle du texte écrit) à une autre (celle de l'image). De ce fait, et inéluctablement, l'image ramène au 
texte écrit, mais ne s'y confond pas, dans la position dialectique que nous venons de lui assigner.

\section{Le rôle de l'enseignant}

On comprendra alors que le rôle que l'enseignant est amené à jouer subisse le contrecoup de cette nouvelle place assignée à l'image. Beaucoup voient aujourd'hui le français apparaitre comme le ciment de toutes les disciplines et voient conséquemment le professeur de français davantage comme un spécialiste du langage, de la capacité à communiquer que comme un enseignant de lettres ou encore de littérature. Cette transversalité de la discipline, qu'elle soit revendiquée ou au contraire regrettée, connaît un nouvel essor avec l'image dont le lien avec la littérature n'apparait pas d'emblée. L'intégration de l'image semble donc aller dans le sens d'un décloisonnement de la discipline et cela d'autant plus que la polysémie constitutive de toute image implique qu'une image ne signifie rien en dehors d'un contexte et d'une démarche interprétative. C'est dire que toute lecture d'images relève d'une pragmatique interprétative, appréhendée à partir d'indices perçus collectivement et de regards portés individuellement. L'image permet de travailler sur la multiplicité du réel, ce qui en fait un outil précieux dans ce que nous appellerons la reconduite au réel.«Être homme, c'est être capable de transfert dans une autre perspective ", cette formule de P. Ricœur est sans doute une des meilleures justifications de la confrontation nécessaire dans la lecture de l'image du « dit d'un seul » à la parole des autres. La voix ou plutôt les voix doivent retrouver une place à l'école dans l'acceptation et la contestation du discours de l'autre. Si ce que nous lisons dans une image dépend de ce que nous sommes et de ce que nous avons appris, alors il est impossible à deux regards différents de voir la même chose. Cette évidence qui pourrait s'appliquer tout autant au texte littéraire, mais qui ne trouve pas dans les formes actuelles de l'expérience scolaire la possibilité de se réaliser, trouve, avec l'image l'opportunité de se construire. La lecture d'image offre à l'enseignant, et surtout aux élèves, la possibilité de construire le conflit interprétatif, sans le poids des normes que la lecture littéraire présuppose.

10 Cette double exigence, épistémologique et éthique, conduit à une mise en scène, une théâtralisation de la parole interprétative. En mettant l'accent sur la parole échangée on replace les sujets au centre de l'analyse, on renvoie la lecture de l'image à une lecture en acte dans laquelle la notion prioritaire devient celle d'altérité. Regarder implique donc une conversion du regard, la prise de conscience que la manière de regarder d'un sujet est le produit d'une éducation, de l'intériorisation de certains schèmes perceptifs, et d'une expérience personnelle. Il faut apprendre à regarder, apprendre donc à parler aux autres. C'est moins l'image qui doit être modifiée par le regard que le regard par elle. Le sujet regardant accomplit une sorte d'« ethnologie de soi-même » et rencontre l'altérité comme chemin de l'identité. Apprentissages collectifs et individuels se conjuguent de fait dans la lecture de l'image, dans cette entrée en possession d'un langage, d'un savoir, d'une technique, dans un enseignement qui ne vise pas autre chose qu'une entrée en possession de soi $^{3}$.

11 Il s'agira alors, d'un point de vue didactique et pour reprendre les termes de Francis Grossmann, de prendre en compte «l'hétérogénéité interprétative au sein de la classe ». L'enjeu est que l'élève apprenne à mettre en attente, à différer des interprétations, à intégrer de nouvelles représentations, à changer même de point de vue. L'image, parce 
qu'elle valorise précisément le voir, et par là même la représentation et le point de vue, induit naturellement ce travail de développement des capacités métatextuelles, précieux pour toute lecture. Elle va ainsi, en raison de la polysémie vers laquelle elle tend par nature, impliquer une sorte de prise de risque inférentielle d'abord, interprétative ensuite. Le regard de l'autre, porté sur le même objet, ou plutôt la confrontation avec le regard de l'autre favorise l'objectivation du sens et des stratégies qui ont amené à le construire. Les divergences interprétatives permettent alors un raisonnement de type abductif ${ }^{4}$ refusant le sens pré-établi ${ }^{5}$. Parler d'abduction est essentiel en ce que cette dernière place au premier plan l'élaboration de l'hypothèse et l'individuation de l'interprétation. La confrontation d'un regard avec les autres regards rend plus attentives et vigilantes la prise d'indices dans l'image et à terme la construction du sens. La posture interprétative devient de ce fait plus active et consciente d'elle-même et de son objet.

12 Ainsi, nulle vérité définitive, nulle adoption du point de vue de Sirius, ne peut être recevable en tant que telle. Au-delà des constantes et des déterminismes (liés par exemple, mais pas seulement, à l'histoire littéraire, à la présence d'invariants techniques et culturels) nécessairement significatifs, il existe bien une lecture singulière qui laisse une place à l'individu, qui relativise l'importance des savoirs encyclopédiques au bénéfice d'une approche herméneutique. L'image apparaît comme un lieu d'épiphanie ou au moins de dialogue. R. Char dans un poème consacré au Prisonnier de La Tour ${ }^{6}$ évoque les yeux des réfractaires de la Résistance brûlés« aux preuves de cette chandelle» qui « réfléchit son sens dans 'leur'condition ». La vérité ici n'est ni intrinsèque à l'œuvre ni intemporelle, elle acquiert sa force d'un contexte de réception particulier : «Reconnaissance à Georges de La Tour qui maîtrisa les ténèbres hitlériennes avec un dialogue d'êtres humains ». Le dialogue est à la fois dans le tableau, dans l'échange du spectateur avec l'image et dans l'échange des spectateurs entre eux. Le rôle de l'enseignant est bien alors de mettre en place les conditions de ce dialogue dans la classe, de renoncer, pour une part à son expertise encyclopédique, de faire jouer les différentes postures interprétatives et les domaines de savoirs qu'elles impliquent.

En cela, la lecture de l'image appelle l'enseignant à se situer dans l'interdisciplinarité et à adopter des postures nouvelles. Pourtant, et de manière totalement paradoxale, la nécessaire maîtrise d'outils d'analyse nouveaux semble induire un nouveau cloisonnement et encourager les attitudes transmissives: à côté de celles de la grammaire, du vocabulaire, de la lecture de textes écrits, littéraires ou non littéraires, apparait une nouvelle rubrique: l'image. Tel manuel de collège (Hachette éducation, 1999, classe de troisième, par exemple) termine systématiquement ses chapitres par une "lecture de l'image " et inclut dans son sommaire une "relation texte - image" absolument pas explicitée par la suite. Certains manuels du primaire ou du collège font de la maîtrise des savoirs savants sur l'image un objectif en soi, exhibant des lexiques de cinéma, produisant des écritures de scenarii, ou questionnant sur les aspects techniques de l'image, reprenant par là les errements d'une dérive techniciste déjà constatée dans l'étude du texte écrit, amplifiée ici par la caution qu'apportent les professionnels du cinéma à ce langage technique. Le rôle de l'enseignant n'est donc pas facilement circonscrit : passeur de sens, tout particulièrement dans la lecture d'image, il doit aussi composer avec des savoirs savants qu'il ne peut ignorer. 


\section{Une nouvelle approche}

14 Que dire alors du sort de la littérature prise au cœur d'une tension entre l'émergence d'un cloisonnement d'activités spécialisées fragmentant le cours de français et l'évidence d'un décloisonnement ressenti comme nécessaire à la cohérence d'une discipline, d'un apprentissage, de la formation d'un sujet? L'image semble ainsi, à son insu d'ailleurs, révéler, au sens photographique du terme, le paradoxe d'une discipline éclatée, par conséquent fragilisée, par des exigences contradictoires auxquelles on lui demande de répondre simultanément. Avec la lecture d'image, il existe tout un espace, à la fois de lecture, d'invention et de distinction (au sens que Bourdieu donnait à ce terme), un espace commun à celui des ateliers d'écriture, des pratiques de lecture diversifiées (lectures partagées notamment), des activités orales (avec le débat) qui œuvrent dans une perspective voisine. L'image peut occuper cet espace-là, par sa capacité à appeler une posture anthropologique. Elle appelle un nouveau mode de lecture appuyé sur une subjectivité assumée à partir d'un travail sur les codes en présence dont l'arbitraire et en même temps la nécessité apparaissent clairement à leurs utilisateurs. Ce nouveau mode de lecture que nous allons tenter de préciser apparaît comme opératoire de la maternelle à l'université en raison de la nature même de l'image et ce n'est pas là son moindre intérêt. L'image dans cette perspective permet de reconfigurer le champ littéraire en faisant réfléchir notamment aux modalités d'autonomisation du discours littéraire comme à la porosité de la frontière établie entre le littéraire et le non-littéraire, questions que pose aussi l'écriture autobiographique (entre le roman autobiographique et le récit de vie, quelle littérarité se joue ?)

15 L'image trouve sa place et sa légitimation scolaires dans ce qui apparait comme une formation du sujet à une médiation culturelle et non dans une instrumentalisation suspecte pour elle-même et pour la littérature. Dans la lecture de l'image, il s'agit donc moins de promouvoir un savoir culturel qu'une compétence lectorale (spectatoriale en réalité) définie comme la capacité à comprendre l'image en acte et non pas comme un objet figé, ossifié. Toute image est dynamique, complexe, contradictoire même et ambiguë. Cette reconnaissance de l'incomplétude de toute lecture de l'image oblige à une certaine prudence. Prudence d'autant plus nécessaire que tout regard s'appuie sur une subjectivité qui en retour exige une démarche d'objectivation. L'introduction de la dimension anthropologique va dans ce sens, en même temps que la reconnaissance du sujet regardant, le « retour de l'acteur » pour reprendre la formule d'Alain Touraine.

16 Le structuralisme et la sémiotique ont mis en avant l'autoréférentialité des œuvres, entendant ainsi apporter une réponse aux dérives interprétatives qui ne voyaient dans les œuvres qu'un pâle reflet du réel. Or cette réponse a conduit à récuser la prise en compte de la référence dans l'analyse et par conséquent à enfermer l'image dans un univers en soi, coupé du monde comme des attentes du lecteur, à réduire l'œuvre à des jeux formels vidés de toute substance ou à la simple duplication du réel. Combattre cet état de fait suppose de nouvelles approches. La lecture de l'image permet la recherche d'un apprentissage qui échappe à la forme académique du commentaire comme à la langue désincarnée de la norme universitaire, sans pour autant que soit abandonnée la rigueur de la recherche. Dans les années cinquante, l'anthropologie a dû faire face à un défi assez proche et la collection Terre Humaine, en particulier, a cherché à rompre avec l'écriture universitaire et sa prétention à la scientificité, au nom précisément de la singularité d'un 
être et d'une culture ${ }^{7}$. L'image est en effet un matériau didactique qui facilite le passage d'une observation traditionnellement objectivante à une "observation participante", celle-la même dont J. Malaurie fait une condition nécessaire du travail anthropologique ${ }^{8}$. Or la prise en compte de l'étrangeté de l'objet d'enseignement et dans le même temps du caractère familier qu'il doit acquérir pour lui fait de l'élève une sorte d'anthropologue en art (à la manière du travail de Levi-Strauss dans Tristes Tropiques). C'est en réintroduisant la sensibilité, l'intériorité, que la critique universitaire a expulsées, que l'on impliquera l'élève dans ce qu'il observe et que l'on fera naître chez lui le désir d'observer et de parler. Car il s'agit bien de redonner une parole à l'élève. Chaque étude, chaque commentaire d'image doit être ainsi le résultat d'un itinéraire personnel, d'une expérience vécue directement et non plus seulement par procuration. Il s'agit selon les termes de J. Malaurie dans son introduction aux Carnets d'enquête de Zola de faire " prendre conscience que l'humble vérité tant recherchée par Maupassant ne peut être atteinte que dans la mesure où l'on vit son observation».

17 Cet accent mis sur l'engagement personnel de l'élève dans l'acte de lecture peut se traduire par une forme plus ou moins explicite de journal de bord tenu partiellement ou totalement et installant la parole de l'élève dans les marges du discours savant. Il s'agit davantage de provoquer la rencontre de l'élève avec l'œuvre et de lui en faire prendre conscience par une relation écrite. La composante dramatique n'est pas forcément absente de ce récit et situe ce dernier à la croisée de la critique et de la littérature. Dans cette perspective la lecture de l'image n'est donc pas statique, mise au service d'une finalité interprétative préconstruite par des discours savants. Elle réinscrit au contraire le voir dans un vivre, dans un cheminement cognitif qui n'apparaît dans toute son évidence qu'à celui qui l'a effectué. C'est d'ailleurs dans la même perspective aujourd'hui que le discours autobiographique tend à être pratiqué à l'école non pas tant comme objet de lecture que comme travail de l'élève sur soi.

Plus précisément, on vise à réhabiliter le subjectif comme approche et le récit comme analyse. Cela implique, du côté de l'élève, de procéder à une analyse d'abord résolument narrative qui l'aide à se réapproprier l'écriture dont il use. 
ASTRE M.-L, DEFRADAS M., ROUSSELOT M. et COLMEZ F. (dir.) : Français $3^{e}$. L'Art de Lire - nouvelle collection. Paris, Larousse-Bordas, 1999, p. 171, 320 pp.

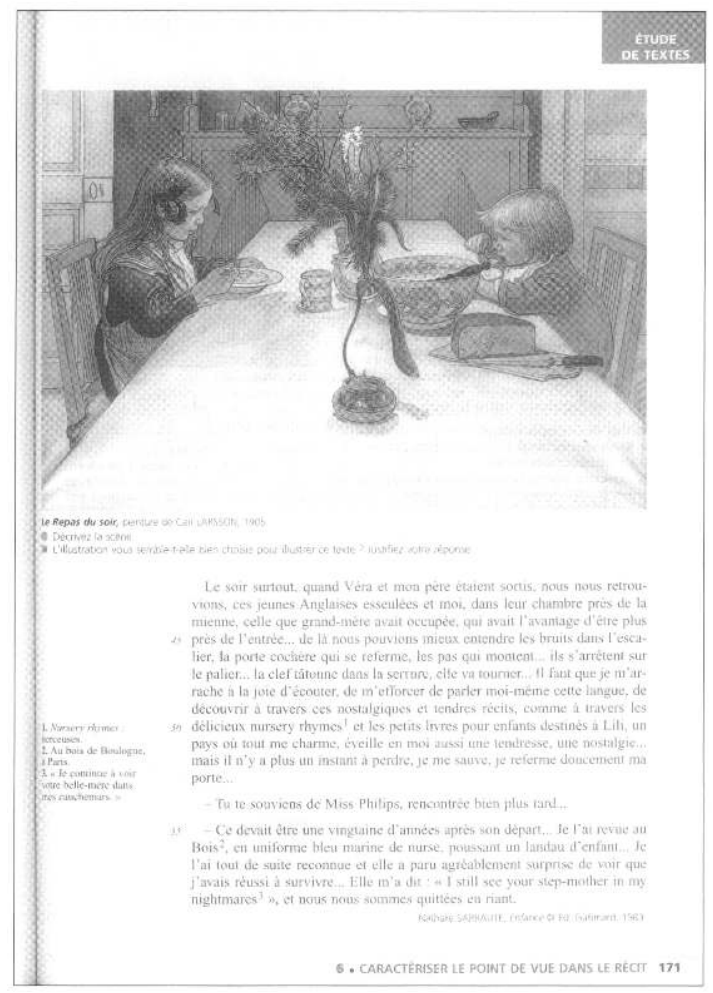

Un exercice pratiqué parfois dans les collèges consiste par exemple à demander aux élèves d'écrire le récit d'un ou plusieurs épisodes de la vie d'un personnage à partir du portrait peint de ce dernier. Les élèves doivent choisir eux-mêmes le portrait, dans une époque précisée par l'enseignant, qui tienne compte de l'histoire des représentations telle qu'elle est présentée dans le cours de français, notamment par le biais des textes littéraires et des textes documentaires étudiés. Il s'agit de permettre à l'élève de se servir de la contextualisation qui lui a été fournie pour arriver à s'exprimer en tant qu'acteur de son propre regard sans pour autant que l'activité de lecture de l'image s'efface ou devienne un simple prétexte. On a là une première forme de commentaire, par l'explicitation narrative d'une interprétation de l'image. Si la lecture de l'élève à ce stade relève davantage d'une dimension affective, il n'en reste pas moins que l'aspect énigmatique forcément donné au tableau - car il s'agit de «deviner » une personnalité, d'en percer le langage muet du reflet - invite l'élève à aller vers une lecture intellectuelle, puisqu'il va construire une lecture à partir d'une image conçue comme signifiante, puisqu'il va exercer son activité en fonction de ce qu'il voit et de ce qu'il sait ou devine.

Elle permet ainsi au moi d'exister dans le rapport qu'il a avec l'autre, de construire son discours sur l'objet au sein d'un dialogue où il a une place réelle. La parole individuelle n'est alors pas prise isolément mais incluse dans une superposition de " témoignages $»^{(9)}$ : ainsi contre tout dogmatisme scolaire apparaît la multiplicité du réel. C'est en ce sens que le rapport à l'autre peut devenir fécond, par le double niveau de lecture induit: d'une part la relation de l'image, d'autre part la manière dont elle est perçue. La lecture d'image autorise plus que toute autre ce retour (détour?) au subjectif en ce que l'élève devant l'image ne peut se transformer en pure instance énonciative mettant à distance son affectivité: la nature même de l'image le lui interdit, nous l'avons vu. Entre 
l'anthropologue et le critique d'art l'élève a une place, sa place. Cela implique du côté de l'enseignant de refuser d'être celui qui sait, de chercher simplement à être celui qui "allume des feux », comme l'écrivait Montaigne.

DARCOS X. et TARTAYRE B. : Le XVII siècle en littérature. Paris, Hachette (Perspectives et Confrontations, 1987 , p. 35,400 pp.

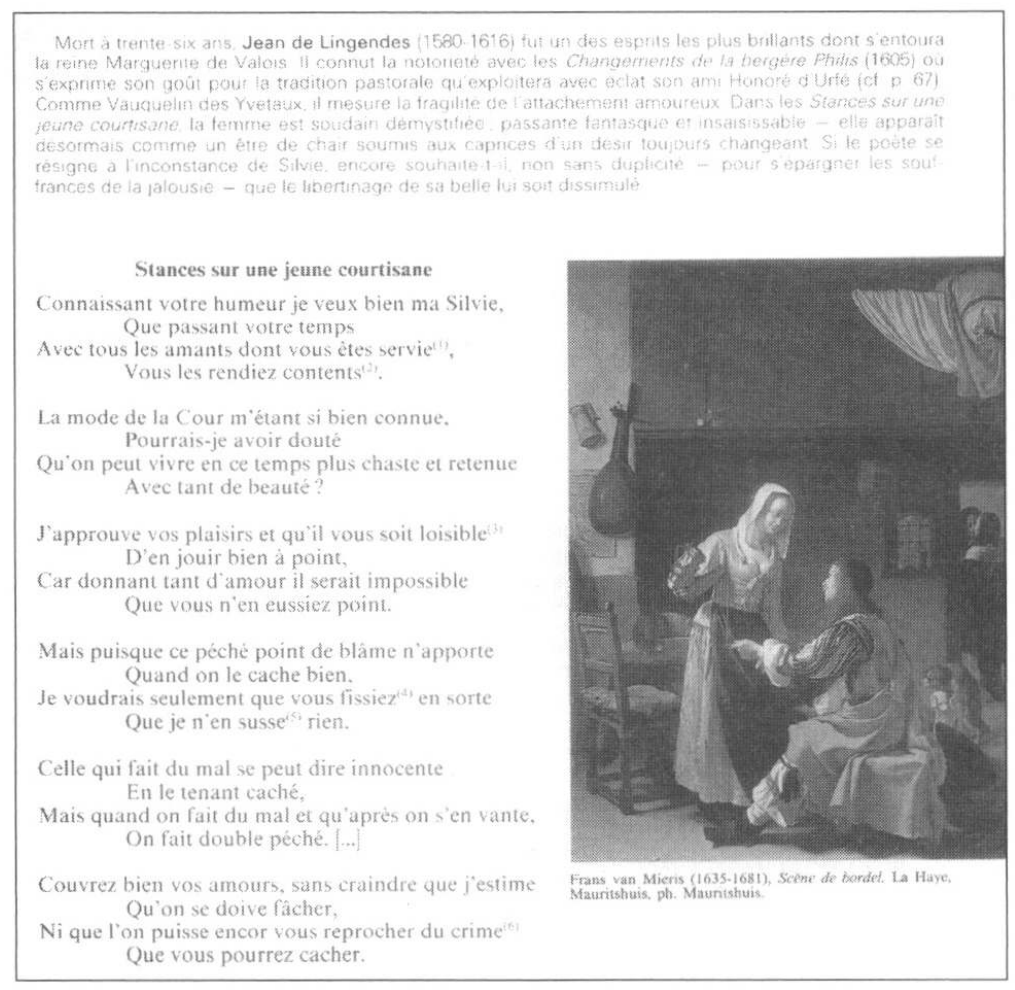

\section{Conclusion}

Notre propos a tenté de réhabiliter la notion de témoignage, de faire de l'observateur acteur qu'est l'élève à la fois le sujet et l'objet de son observation, de rendre en somme une parole, sinon la parole, à l'élève. Il s'agit bien de réintroduire dans l'approche littéraire et toutes les activités de lecture, à partir de la lecture d'image, la sensibilité, l'intériorité qu'une méthodologie excessive a fait disparaître. Cette immersion intellectuelle et émotionnelle recherchée vise à rendre le lecteur familier de l'œuvre d'art, c'est-à-dire à le faire entrer dans un "rapport sans dépendance ", celui-là même dont parle M. Blanchot ${ }^{10}$, familier de soi et familier de l'autre en le laissant être, dans la licence de sa parole sans prétendre l'arraisonner ni l'arrimer à une quelconque raison didactique. Il s'agit aussi dans le même temps de faire que le monde de l'art ne s'oppose plus au monde de la vie quotidienne comme le sacré au profane.

Nous avons évoqué l'image dans ses aspects généraux, artistiques le plus souvent, et l'élève de manière tout aussi globale. Notre propos était de souligner les enjeux formateurs de la lecture d'image, dans le cadre restreint de cet article, et ceci explique cela. Pour autant, il est clair que toute image ne vaut pas une autre, que la photographie de presse et le tableau médiéval ne suscitent pas les mêmes possibilités de lecture. Un chantier nécessaire consiste alors à identifier les usages didactiques des différentes 
images disponibles, de faire, non pas une typologie idéale des images, mais bien un répertoire raisonné des formes iconiques dans une perspective didactique. Quant à l'élève, si l'approche anthropologique que nous préconisons, parce qu'elle nous semble répondre aux ambitions des nouveaux programmes et garantir une véritable dimension formative, constitue le modèle de référence à mettre en œuvre, il est clair, aussi, qu'elle ne saurait se décliner uniformément. Différentes variables éducatives conditionnent sa réalisation: l'âge des élèves, les situations scolaires particulières, ou les conditions matérielles pour approcher l'image sont, parmi d'autres, des paramètres à prendre en compte.

Ces réserves établies, il reste que lire une image mène à une forme de lecture créative dans laquelle seront donnés du sens et des histoires aux images. Bien sûr, beaucoup de choses échappent à l'élève en raison, en particulier, de la nature protéiforme des symboles mais n'est-ce pas le propre de l'expérience que de ne pas être formulable en mots ? ${ }^{11}$ Ce faisant, l'image devient le lieu de l'interprétation retrouvée, un lieu où se prouve et s'éprouve la littérature. Par la particularité de son objet, la lecture - qu'elle soit d'images ou de textes écrits - relève ainsi d'une démarche proprement " herméneutique » ${ }^{12}$ en ce qu'elle apparaît non pas fondée sur l'établissement de règles, dont l'apprentissage et l'usage seraient donc systématisés, mais sur l'interprétation et le dialogisme qu'elle implique. C'est bien alors l'activité du sujet, fondatrice de l'acte de lecture, qui est mise en avant et son identification qui permet de retrouver le travail des subjectivités à l'œuvre dans le groupe social. Que la lecture d'image aide à penser cela dans l'école, et à en faire profiter toute expérience de lecture, suffit à lui donner toute sa légitimité.

\section{NOTES}

1. "L'image n'est pas tenue pour une illustration accessoire, mais pour un objet d'analyse, dont l'étude permet, sur le plan pédagogique, d'éduquer le regard, d'enrichir la sensibilité, de stimuler l'imagination, d'éveiller l'esprit critique et de développer l'expression »,Bulletin Officiel, № 25, 22 juin 1995.

2. Nous ne nous prononçons pas ici sur la validité des lectures ainsi proposées.

3. Voir l'analyse de l'acte de celui qui «comprend» men2e par M. Henry dansLa Barbarie, Grasset, 1987.

4. L'abduction, telle qu'elle est définie par W.S. Peirce, consiste a prélever ailleurs que dans le champ du phénomène observé la Loi qui va permettre l'explication. C'est cette abduction qui caractérise souvent par exemple, dans le récit d'énigme surtout, la démarche argumentative du détective, ce qu'on appelle sa méthode ou encore son flair. Ce type de raisonnement permet d'exprimer et de développer une certaine fantaisie, patente dans certains discours explicatifs de détectives.

5. Voir les travaux à ce sujet de H. Parret, 1999.

6. Feuillets d'Hypnos. Paris, Gallimard, 1946.

7. Voir à ce propos le bel ouvrage que $\mathrm{P}$. Auregan a consacré a ce sujet : Des récits et des hommes. Paris, Nathan - Plon, 2001.

8. Idée déjà présente chez Malinowski :Les Argonautes du pacifique occidental. Paris, Gallimard, p. 63 
9. Quasiment au sens chrétien du terme.

10. L'Amitié.Paris, Gallimard, 1971, pp. 328-329.

11. Voir à ce propos la parole de l'Ecclésiaste citée par A. Manguel : «Toutes choses sont en travail au-delà de ce qu'on peut dire; l'œil n'est pas rassasié de voir, et l'oreille ne se lasse pas d'entendre. ",inUn Livre d'images, trad. Française. Arles, Actes Sud, 2001.

12. Rappelons qu'en philosophie l'herméneutique contemporaine s'est développée en France avec P. Ricœur et en Allemagne avec H.G. Gadamer, par son refus notamment de la réduction positiviste de la vérité au modèle "deductif-nomologique » des sciences expérimentales de la nature.

\section{AUTEUR}

\section{FRANÇOISE DEMOUGIN}

Université Stendhal-Grenoble III, Équipes Didaxis et Ceditel 